\title{
Study on the Influencing Factors of Chengdu Residents' Travel Decision-Making to Thailand: Take the Tourists' Perception of Thailand Travel Blogs From Mafengwo Website as an Example
}

\author{
Hui HE ${ }^{\mathrm{a}}$ Heng YANG ${ }^{\mathrm{b},{ }^{*}}$ and Tong XIANG ${ }^{\mathrm{c}}$ \\ College of Tourism,Sichuan Agricultural University, Chengdu, Sichuan,610039,China \\ ah952446491@163.com, b21834566@qq.com, '2637564633@qq.com
}

Keywords: travel blogs, travel decision-making, quality analysis

Abstract. This paper takes Chengdu residents as the object of investigation, to explore which contents of travel blogs influence their travel decision-making to Thailand. By recruiting 43

Chengdu residents as research objects, this paper analyzes the contents of blogs that affect their travel decisions after reading 5 Thailand travel blogs and relevant questionnaires. Finally, through the questionnaire of Chengdu residents, the content of the online travel information mainly affecting Chengdu residents' travel to Thailand is obtained. Conclusions are: Tourists think that the most influential content of travel blogs for their decision-making is tour routes and itinerary arrangements, followed by traffic information, safety precautions, fees, cultural customs, accommodation information, food and beverage information, etc. tourists are more interested local specialties in food and beverage information, and tourists also show great concern about the description of the characteristic culture.

\section{Introduction}

Data from the Ministry of Culture and Tourism of the People's Republic of China in 2018 show that the number of Chinese outbound tourists reached 149 million, an increase of $14.7 \%$ over the same period last year[1]. China's outbound tourism market is developing rapidly, ranking first in the world's outbound tourism. With the continuous development of network technology, more and more people travel abroad to book hotels and flights, collect travel information oline[2]. The popularity of mobile Internet enables tourists to quickly grasp the information of tourist destinations, and travel blogs has also emerged. Travel blogs are texts published by tourists to describe their travel process and feelings on the Internet based on their own travel experience[3]. By using interpersonal influence media such as travel blogs, tourism companies can gain a competitive advantage[4]. From the perspective of travel blogs, this paper discusses its influence on outbound tourists' decision-making, which is not only helps the relevant tourism enterprises to carry out the marketing of tourism products, but also can provide some suggestions for government departments to provide better policy services.

\section{Literature Review}

At present, the research on travel blogs at home and abroad mainly focuses on the image perception of tourist destinations [5-7], the network structure of tourism flow [8-10]and the analysis of the role of tourism marketing[11-13]. Among them, the construction of the image of tourist destination based on the travel blogs is the focus of scholars in recent years. For example, Wang Lina and Li Hua analyzed the contents of the English travel blogs of foreign tourists, and used the method of rooted theory to analyze the logical relationship between the sources of negative perception of the image of Chinese tourist destinations. Travel blogs is a way to tell the tourist's travel experience in a story-telling way, and this kind of story-rich blog can promote the potential tourists to become real tourists[14]. Some scholars point out that tourists attach great importance to the traffic, accommodation facilities, food and catering in the contents of the travel blogs, and have a greater impact on the behavior of travel decision-making[3]. However, the relevant research are still lacking, the research on the tourist behavior under the travel blogs needs to be further deepened. 
Throughout the research on travel blogs at home and abroad, the objects of the study are more inclined to a single network text, the tourist destination seldom focuses on a certain country or region, and studies also rarely break down outbound travel methods. This paper mainly starts with the travel blogs of specific tourist destination countries - Thailand, based on the specific potential tourist - Chengdu residents' perception of the ravel blogs, combing the tourist perception with simple network text analysis method to analyze the impact of different contents of the travel blogs on the decision-making of tourists, so as to provide more scientific guidance on the product portfolio and marketing strategy of tourism enterprises.

\section{Research Methodology}

\subsection{Data Collection}

This study recruited 43 Chengdu residents as research subjects.In order to carry out more targeted research, this paper focuses on Chengdu, sichuan province. According to the 2018 big data report on outbound Chinese tourists released by the China tourism academy and ctrip, Chinese people spent 120 billion us dollars overseas in 2018. "New first-tier" cities have contributed the largest number of new customers to the outbound tourism market, among which chengdu is the fourth outbound tourism departure city in China. we chose potential outbound tourists in Chengdu as the research object, which is relatively representative.

\subsection{Specific Implementation Scheme}

Thailand, Japan, Malaysia, Vietnam, the United States, Indonesia, the Philippines, Singapore, South Korea and Australia have become the most popular overseas destinations for Chinese tourists, according to the big data report on China's outbound independent travel in 2018 released by Mafengwo website. This paper selected Thailand, the first outbound tourism destination, as the destination country of this study, and selected 5 travel blogs of Thailand with the highest comprehensive rating from the Mafengwo website to download and sort them out as the research materials. The selection criteria include the number of views, comments and so on. The travel blogs almost cover more than $95 \%$ of Thailand's hot spots.

\subsection{The Implementation of This Study is Mainly Divided Into Three Steps}

Step 1: select the research materials. download 5 travel blogs of Thailand with the highest comprehensive score on the Mafengwo website and systematically organize them for future research.

Step 2: select the subjects to browse travel blogs in a given situation and complete relevant operations. The specific situation prompt is: suppose you recent time and money are relatively abundant, so have the idea of traveling abroad. The destination is not certain, but Thailand is in your consideration. As it is the first time for you to travel abroad, you still want to make some strategies in advance, so you plan to refer to the travel blogs published on the Mafengwo website to make a travel decision. At the same time, the study need each participant to mark the contents that could influence their travel decisions or make you interested and impressed.

Step 3: questionnaire survey. After completing the above steps, the subjects need to fill in a questionnaire about this study.At the same time, in order to further verify the conclusion of the study, the conclusion formed by qualitative analysis of the marked travel blogs was used as the item of the questionnaire for residents in Chengdu.

\subsection{Data Analysis and Processing}

The first phase of the investigation lasted for 11 days from October 10, 2019 to October 20, 2019. 215 pieces of travel notes and 43 questionnaires were distributed, and 178 pieces of marked travel blogs and 43 questionnaires were finally recovered. Firstly, the recovered 178 travel notes were processed with Word, and the unmarked text content was removed. Then Nvivo11.0 software was used to encode and analyze the text marked contents of the recovered 178 travel blogs.In the second phase of the survey, 172 questionnaires were distributed to residents in Chengdu in November 2019, 
and 135 valid questionnaires were obtained after excluding those who had not read travel blogs from Thailand. Statistical software SPSS 22.0 was used to process and analyze the data.

\subsection{Statistical Characteristics Analysis of the Investigated Objects}

In this study, female respondents were slightly more than male respondents, accounting for $55.81 \%$ and $44.19 \%$ respectively. In terms of age structure, there are more young and middle-aged people between 23 and 50 years old, accounting for $62.79 \%$ of the total. These people have a higher income level, which is an important group for online search and the main body of tourism consumption.

\section{Results}

Nvivo11.0 software was used to conduct word frequency statistics and semantic analysis on the tagged travel blogs. Through open coding, obtaining a total of 23 first-level nodes. Then, the open coding results were clustered simply through logical relations to form the spindle coding results. Finally, the spindle coding results were classified again through literature support and practical experience to form the secondary spindle coding results. Table 1 shows the main three categories of spindle coding process of tourism information that affect tourists' decision to visit Thailand.

Table 1. Information from Travel Blogs Spindle Coding Process.

\section{Part of the Original Material}

The First

The Second

The Third Order Concept

Order Concept

Order Concept

According to your personal situation, choose to apply for a tourist visa or lading visa in advance.

It's important to note that booking a domestic flight to Thailand, The key is to buy checked baggage in advance

Download the taxi-hailing app "grap" from the appstore. After registering an account, you can pay by alipay in Thailand.

Visa

Flight booking

Airport

Transportation

Before the Trip

On the Trip

Traffic Information

On the Trip

The main tower is the most famous, here is one of the scenery of 86 edition [journey to the west] !

If you want to take public transportation, you should leave a

lot of time on the road, especially not during rush hours

The triangular wing flight booked in the morning
Less

\begin{tabular}{ccc} 
Well-known & Spots & \\
Spots & Shopping & Tour Routes \\
Popular Spots & Schedule & and Itinerary \\
Schedule & Entertainment & Arrangements \\
Entertainment & Project & \\
Project & & \\
\hline
\end{tabular}


It is said that April to October is the rainy season in Thailand every year, and October to April is the very hot summer, so no matter when you go, please prepare enough summer clothes.

when passing the customs in Thailand, it is recommended that two people exchange $2 \mathrm{~W}$ thai baht and carry $4 \mathrm{~K}$ RMB

The insurances I would recommend:Traffic accident, flight delay, item lost, accidental injury/death, medical

The Weather

Currency

The Entry and Exit

Insurance

Others
The Weather

Currency

The Entry and

Exit

Safety Precautions

Insurance

Others treatment/medical delivery, third party liability

By spindle coding, we found that the respondents after browsing the blogs thinks that the several factors that can strongly affect their travel to Thailand are tour routes and itinerary arrangements, traffic information, safety precautions, cultural customs, fees, accommodation, food and beverage information, but the influence of comments and language is relatively small.The above conclusions are also validated by the questionnaire statistics from 43 respondents and 135 chengdu residents

( Table 2).

Table 2. Influence Factor Statistics from Questionnaire statistics from 135 chengdu residents.

\begin{tabular}{cccccc}
\hline $\begin{array}{c}\text { Influence } \\
\text { Factor }\end{array}$ & Traffic & Accommodation & Food and Beverage & $\begin{array}{c}\text { Tour Routes and } \\
\text { Itinerary }\end{array}$ & $\begin{array}{c}\text { Safety } \\
\text { Precautions }\end{array}$ \\
Mean & 4.36 & 4.36 & 4.36 & 4.36 & 4.36 \\
\hline $\begin{array}{c}\text { Influence } \\
\text { Factor }\end{array}$ & $\begin{array}{c}\text { Cultural } \\
\text { Customs }\end{array}$ & Fees & Language & Comments & $\begin{array}{c}\text { The Impact of } \\
\text { Publishing } \\
\text { Platforms }\end{array}$ \\
Mean & 4.23 & 4.23 & & & 4.23 \\
\hline
\end{tabular}

\subsection{The Influence of Travel Routes and Itinerary on Travel Decision-Making}

Among all the information affecting tourists' decision-making, travel routes and itinerary arrangements ranked the first, with reference point of 744. Meanwhile, in the open-ended question of "what is the most impressive thing about all travel blogs?", most respondents talked about the arrangement of tour routes. The reference point of scenic spots is 410 , which also includes popular scenic spots and less well-known scenic spots. Tourists are especially interested in the description of "grand palace", "zheng wang temple", "reposing Buddha" and "night market". A few people also prefer some less well-known scenic spots. This part of respondents mainly are college students. "What impressed me most was the scenic spots in travel blog 4, especially the popular animal home stay," was the answer given by one of the respondents to the question "what is the most impressive thing about all the travel blogs?".

\subsection{Influence of Traffic Information on Travel Decision-Making}

Transportation information includes the arrangements before and during the trip. The reference point before the trip is 228 , which mainly includes visa, air ticket reservation and airport pickup. Studies have shown that visa convenience can indeed promote outbound tourism of a country or region [15]. During the coding process, it is found that tourists pay special attention to the information of "visa" and "landing visa", including visa cost, procedures and precautions, etc. The reference point that tourists pay attention to traffic information during their journey in Thailand is 316 , accounting for $58.09 \%$ of the total traffic information. The most attention are grab app, tutu car, 
car rental, and drive yourself. Outbound tourists will pay close attention to the traffic information of the tourist destination and take corresponding tourism actions before making tarvel decisions.

\subsection{Influence of Safety and Precautions on Travel Decision-Making}

Safety and precautions also have a greater impact on the decision-making process of outbound tourism. The total reference point is 461 , which is second only to tour routes and itinerary arrangements and traffic information. It is found that tourists pay special attention to the information of entry and exit, including how to fill in the entry and exit card, entry and exit notice and so on. At the same time, tourists also pay great attention to weather information, such as "sun protection", "hot", "mosquitoes" and so on. Meanwhile, tourists also pay close attention to the contents about "currency exchange", "exchange rate", "insurance". After Chinese tourists in Thailand ran into dozens of serious accidents, such as the island of phuket tourists capsized accident, and frequent traffic accidents, Outbound travelers will worry more about their travel safety than domestic travelers, so safety problem for outbound tourists' decision-making played a large role.

\subsection{Influence of other types of Tourism Information on Travel Decision-Making}

The reference point of the cost is 347. Outbound travel will go farther than domestic travel, which makes transportation more expensive. Therefore, outbound tourists will pay more attention to the cost of the whole trip before making travel decisions. The reference point of cultural customs is 300. "I have always wanted to go to Thailand for its exotic culture and island-style. These travel blogs gave me some ideas for my plan," one of the respondents said in the open-ended question of "what impressed you most about all the travel blogs?"The reference point of accommodation information is 268. Tourists will not only pay attention to the accommodation environment, transportation, price and so on, but also pay attention to how to book and the experience of passing tourists. At the same time, local cuisine information is more attractive for potential tourists and language and comments information have less impact on outbound tourists' decision-making.

\section{Conclusion}

This study found that tourists concern more about travel routes and itinerary arrangements, traffic information, safety precautions, cultural customs, fees, accommodation, food and beverage information, and pay less attention to language, comments, blogger and publishing platform.

The research mainly puts forward the following suggestions for the operators of tourism enterprises: User-generated Content (UGC) should be valued and utilized, especially for the middle-aged and young people. Improve the existing online tourism database, according to the interests and needs of tourists. Tourist destination, OTA cooperation platform, travel agents, and so on, should actively cooperate and build a more perfect tourism information system, to get the tourists' real-time demand data. We should combine the tourist demand with the development of tourism projects and products, to offer visitors a more innovative and experiential travel plan.

\section{References}

[1] Information on http://zwgk.mct.gov.cn/auto255/201902/t20190212 837271.html?keywords=

[2] Jinsong Yang. China Outbound Tourism Development Report 2019[R]. 2019.

[3] Wei Cheng, Lina Sui, Yuzhu Zhang. Analysis of the Influence of Online Travel Blogs on Tourists' Travel Decisions[J]. Resource Development and Market, 2016, 32(3): 365-368.

[4] Litvin S.W.-Goldsmith-R.E.-\&PanB. Electronic word-of-mouth in hospitality and tourism management[J]. Tourism Management, 2008, 29(3): 458-468.

[5] Qing Feng, Yijun Tian, Gennian Sun. A Study on Image Perception of Shaanxi Tourist Destinations based on Travel Blogs -- A Case Study of Eight 5A Tourist Attractions in Shaanxi Province[J]. Resource Development and Market, 2018, 34(11): 1623-1628. 
[6] Hua Qu , Yankun Liang . Study on Image Perception of Tourist Destination in Guangzhou based on Travel Blogs[J]. Guangdong Social Science, 2017,(6): 204-211.

[7] Tseng Chi,Wu Bihu,Morrison Alastair-M,et al. Travel Blogs on China as a Destination Image[J]. Elsevier Ltd, 2015, 46: 347-358.

[8] Yajuan Li, Hongchun Zeng, Chaoran Li, et al. Study on the Tourism Flow Network Structure and Flow Pattern in Mountainous Ethnic Areas -- A Case Study of Enshi Prefecture[J]. Resources and Environment of the Yangtze River Basin, 2019, 28(7): 1612-1622.

[9] Jiang Wu, Lingling Wei, Xing Ji, et al. Spatial Distribution Pattern of Tourism Flow in Gansu Province based on Travel Blogs[J]. Journal of Northwest Normal University (Natural Science Edition), 2018, 54(6): 75-81.

[10] Xianxian Zhang, Jinghan Li, Ying Zuo, et al. Analysis of Spatial and Temporal Behavior Characteristics of Tourists based on Digital Footprints -- A Case Study of Nanjing City[J]. Economic Geography, 2018, 38(12): 226-233.

[11] Burgess s Sellitto-C-Cox-C-et-al. User-Generated-Content (UGC) in Tourism: Benefit and Concerns of Comsumers[A][C]. Paper Presented at the 17th European Conference on Information Systems.2019.

[12] Li Pan. Brand Personality Correspondence Analysis of Mountain Tourism in China[J]. Journal of Sichuan Normal University (Social Science Edition), 2018, 45(2): 75-83.

[13] Volo Serena. Bloggers' Reported Tourist Experiences: Their Utility as a Tourism Data Source and Their Effect on Prospevtive Tourists[J]. Journal of Vocation Marketing, 2010, 16(4): 297-311.

[14] Guoqing Wu. Selection of Risk Perception Dimension of Outbound Tourism for Urban Residents -- A Case Study of Shanghai[J]. Areal Research and Development, 2017, 36(1): 111-116.

[15] Xiangyan Liu, Yiyi Jiang, Xingyang Lv, et al. Influence of Visa Convenience on Outbound Tourism -- an Empirical Analysis based on Panel Data[J]. Journal of Tourism, 2018, 33(12): 46-52. 